J. Austral. Math. Soc. 20 (Series A) (1975), 242-244.

\title{
A NOTE ON LINEAR RECURSIVE SEQUENCES
}

M. MIGNOTTE

(Received 1 April 1974)

Communicated by E. S. Barnes

\section{Introduction}

We consider linear recursive sequences of integers not all zero such that

$$
u_{n+k}=a_{1} u_{n+k-1}+\cdots+a_{k} u_{n}, \quad \text { for } n=0,1,2, \cdots,
$$

where the $a_{j}$ are rational integers.

If

$$
X^{k}-a_{1} X^{k-1}-\cdots-a_{k-1} X-a_{k}=\prod_{j=1}^{h}\left(X-\omega_{j}\right)^{r j}
$$

is the decomposition of the associated polynomial $P,\left|\omega_{1}\right| \geqq \cdots \geqq\left|\omega_{h}\right|$, it is well-known that $u_{n}$ is given by

$$
u_{n}=\sum_{j=1}^{h} P_{j}(n) \omega_{j}^{n},
$$

where $P_{j}$ is a polynomial of degree $<r_{j}$, with coefficients in $Q\left(\omega_{1}, \cdots, \omega_{h}\right)$.

We recall first a theorem of Mahler (1969).

TheOREM $A$. Suppose that the $u_{n}$ are given by (1), where

$$
k=2, a_{1}^{2}+4 a_{2}<0, a_{2} \leqq-2,\left(a_{1}, a_{2}\right)=1 .
$$

Let $\varepsilon>0$ be an arbitrary constant. Then, as soon as $n$ is sufficiently large,

$$
\left|u_{n}\right| \geqq\left|\omega_{1}\right|^{(1-\varepsilon) n} \text {. }
$$

Our aim is to prove the following result.

THEOREM 1. Let $\left(u_{n}\right)$ be a sequence of integers satisfying (1). Suppose that $P$ has at most 3 roots of greatest modulus and that these roots $\omega_{1}, \cdots, \omega_{l}$ are 
simple. Then, there exist $n_{0}$ and $c$, which are calculable, such that, for $n \geqq n_{0}$, we have

$$
\left|u_{n}\right| \geqq\left|\omega_{1}\right|^{n_{n}-c} \text { if } v_{n}=P_{1} \omega_{1}^{n}+\cdots+P_{l} \omega_{l}^{n} \neq 0, \quad l \leqq 3 .
$$

(The polynomials $P_{1}, \cdots, P_{l}$ are constant.)

It is clear that this result is nearly the best possible. It seems to be difficult to extend this theorem to the general case.

\section{A lemma}

LEMMA. Let $x_{n}$ be defined by

$$
x_{n}=b+b_{1} y_{1}^{n}+\bar{b}_{1} \bar{y}_{1}^{n}
$$

where $b_{1}, y_{1}$ are algebraic numbers, $\left|y_{1}\right|=1, b=0$ or 1 . Then, there exists calculable $n_{0}$ and $C$ such that, for $n \geqq n_{0}$, the following implication holds

$$
x_{n} \neq 0 \Rightarrow\left|x_{n}\right| \geqq n^{-c} \text {. }
$$

Proof. Because of $\left|x_{n}\right| \geqq b-2\left|b_{1}\right|$, it suffices to consider the case $b$ $\leqq 2\left|b_{1}\right|$. Put

$$
b_{1}=\left|b_{1}\right| e^{i \psi}, y_{1}=e^{i \theta},\left|b / b_{1}\right|=-2 \cos \phi, \theta, \phi, \psi \in[-\pi, \pi[.
$$

We have

$$
\left|x_{n}\right|=4\left|b_{1}\right|\left|\sin \frac{\psi+n \theta+\phi}{2} \sin \frac{\psi+n \theta-\phi}{2}\right| .
$$

The inequality $\left|x_{n}\right| \leqq \eta$ implies

$$
\left|\sin \frac{\psi+n \theta+\phi}{2} \sin \frac{\psi+n \theta-\phi}{2}\right| \leqq \frac{\eta}{4\left|b_{1}\right|} .
$$

If $\phi \neq 0$, (3) leads to an inequality of the form

$$
|n \theta+m \pi \pm \phi+\psi| \leqq c_{1} \eta, m \in \mathbb{Z},|m| \leqq n, \text { if } \eta<\eta_{0},
$$

whereas, if $\phi=0$, it implies

$$
|n \theta+m \pi+\psi| \leqq c_{2} \eta^{\frac{1}{2}}, \text { if } \eta<\eta_{1} .
$$

In both cases, for $x_{n} \neq 0$, we get

$$
0<|n \theta+m \pi+\psi \pm \phi| \leqq c \eta^{\frac{1}{2}} \text {, if } \eta<\eta_{2} \text {. }
$$

Here $i \theta, i \pi, i \phi, i \psi$ are values of logartithms of algebraic numbers and the conclusion follows from Baker's theorem (1972):

THEOREM. Let $\beta_{1}, \cdots, \beta_{k}$ be fixed algebraic numbers. There exists a calculable constant $C_{0}$, such that for $0<\delta<\frac{1}{2}$, the inequalities 


$$
0<\left|b_{1} \log \beta_{1}+\cdots+b_{k-1} \log \beta_{k-1}-\log \beta_{k}\right|<\delta^{c_{0}} e^{-\delta B}
$$

have no integer solutions $b_{1}, \cdots, b_{k-1}$, with $\max \left|b_{i}\right| \leqq B$.

Here, for $B>2 C_{0}$, we choose $\delta=C_{0} / B$, thus, if $\left|b_{1} \log \beta_{1}+\cdots\right| \neq 0$,

$$
\left|b_{1} \log \beta_{1}+\cdots\right|>\left(\frac{C_{0}}{e}\right)^{c_{0}} B^{-c_{0}} \text {. }
$$

\section{Proof of the Theorem}

We may write

$$
\left|v_{n}\right|=a\left|\omega_{1}^{n} x_{n}\right|,
$$

where $x_{n}$ verifies the hypothesis of the lemma. The conclusion follows at once from the lemma (use (2)).

\section{References}

A. Baker (1974), 'A sharpening of the bounds for linear forms in logarithms', Acta Arith. 21, 117-129.

K. Mahler (1966), 'A remark on recursive sequences', J. Math. Sci. 1, 12-17.

Université Paris - Nord

Place du 8 mai 45

93. Saint Denis (France). 